University of Wollongong

Research Online

Faculty of Informatics - Papers (Archive)

Faculty of Engineering and Information

Sciences

$12-12-2006$

\title{
Phase Unwrapping of Self-mixing Signals Observed in Optical Feedback Interferometry for Displacement Measurement
}

\author{
Lu Wei \\ University of Wollongong \\ Jiangtao Xi \\ University of Wollongong, jiangtao@uow.edu.au \\ Yanguang Yu \\ Zhengzhou University, yanguang@uow.edu.au \\ Joe F. Chicharo \\ University of Wollongong, chicharo@uow.edu.au
}

Follow this and additional works at: https://ro.uow.edu.au/infopapers

Part of the Physical Sciences and Mathematics Commons

\section{Recommended Citation}

Wei, Lu; Xi, Jiangtao; Yu, Yanguang; and Chicharo, Joe F.: Phase Unwrapping of Self-mixing Signals Observed in Optical Feedback Interferometry for Displacement Measurement 2006.

https://ro.uow.edu.au/infopapers/520 


\title{
Phase Unwrapping of Self-mixing Signals Observed in Optical Feedback Interferometry for Displacement Measurement
}

\author{
Abstract \\ With an Optical feedback interferometry (OFI), a self-mixing signal (SMS) can be observed and employed \\ to determine parameters of the semiconductor laser and metrological quantities of an object. In the \\ cases of the measurement of the movement trace of an external target, phase unwrapping of the SMS \\ must be performed, which remains a challenging issue. We report a technique for achieving phase \\ unwrapping of the SMS signals as follows. Firstly, the behaviour of the phase equation of the OFI is \\ studied, based on which general laws for laser phase change with respect to external target movement is \\ extracted. Secondly, the correlation between the laser phase change and SMS signal is studied. Finally \\ phase unwrapping algorithm is developed based on the relationship. In order to make sure that the \\ proposed approach works well in the cases of noisy SMS data, pre-processing algorithms are also \\ discussed. The proposed techniques have been tested by both computer simulation and experimental \\ data which show that excellent agreement can be achieved and the movement trace of the external target \\ can be retrieved.

\section{Disciplines} \\ Physical Sciences and Mathematics

\section{Publication Details} \\ This paper was originally published as: Wei, L, Xi, J, Yu, Y \& Chicharo, J, Phase Unwrapping of Self-mixing \\ Signals Observed in Optical Feedback Interferometry for Displacement Measurement, International \\ Symposium on Intelligent Signal Processing and Communications, 2006 (ISPACS '06), Yonago, Japan, \\ 12-15 December 2006, 780-783. Copyright 2006 IEEE.
}




\title{
Phase Unwrapping of Self-mixing Signals Observed in Optical Feedback Interferometry for Displacement Measurement
}

\author{
Lu Wei*, Jiangtao $\mathrm{Xi}^{*}$, Yanguang $\mathrm{Yu}^{\dagger}$, Joe Chicharo* \\ *School of Electrical, Computer and Telecommunications Engineering, University of Wollongong, Northfields Ave, \\ Wollongong, NSW, 2522, Australia \\ Corresponding Author, Tel: +61-2-4221-3412, Fax: +61-2-4221-3236 \\ E-mail: jiangtao@uow.edu.au \\ 'Department of Electronic Engineering, College of Information Engineering, Zhengzhou University, \\ Zhengzhou 450052, China \\ Email: yanguangyu@zzu.edu.cn
}

\begin{abstract}
With an Optical feedback interferometry (OFI), a self-mixing signal (SMS) can be observed and employed to determine parameters of the semiconductor laser and metrological quantities of an object. In the cases of the measurement of the movement trace of an external target, phase unwrapping of the SMS must be performed, which remains a challenging issue. We report a technique for achieving phase unwrapping of the SMS signals as follows. Firstly, the behaviour of the phase equation of the OFI is studied, based on which general laws for laser phase change with respect to external target movement is extracted. Secondly, the correlation between the laser phase change and SMS signal is studied. Finally phase unwrapping algorithm is developed based on the relationship. In order to make sure that the proposed approach works well in the cases of noisy SMS data, pre-processing algorithms are also discussed. The proposed techniques have been tested by both computer simulation and experimental data which show that excellent agreement can be achieved and the movement trace of the external target can be retrieved.
\end{abstract}

\section{INTRODUCTION}

The optical feedback self-mixing (OFSM) phenomenon has been studied extensively in the past two decades and has seen its applications in the measurement of the metrological quantities such as vibration, displacement and velocity of a moving object [1-4]. OFSM occurs when a small portion of the laser beam emitted from a semiconductor laser (SL) is reflected or backscattered by an external target and re-enters the laser cavity, resulting in variance of the lasing field in terms of both frequency and amplitude. Therefore, by intentionally imposing movement to the target and picking up the laser intensity fluctuations with a photodiode at the rear of the SL, the metrological quantities regarding the external target as well as the parameters of the SL can be measured $[3,5]$.

The target displacement has been measured with a fringe-counting technique in the moderate feedback regime $(1<\mathrm{C}<4,6)$ where the interferometric waveform is sawtooth like and exhibits hysteresis in the work [2] with a resolution of $\lambda / 2$. Later efforts were attributed to improve the measuring accuracy of this technique. Addy et al. [6] improved resolution up to $\lambda / 4$ by a misalignment of the reflector with $\mathrm{C}>1$ and a mirror as a target. Bosch et al. [7] developed an algorithm based on the interpretation of the interfringe and of the fractional fringe to linearize the measured displacements with a rough target. In [8,9], a fast modulation of the interferometer phase was generated by means of LD current modulation, resulting in the LD wavelength shift $\Delta \lambda$. By properly sampling the SM signal synchronously with the dither, the resolution is increased to $\lambda / 10$.

The present work starts from analysis of the mathematical model of the OFSM system. The target displacement (or target movement track) is computed from the model with the aid of a novel phase unwrapping technique. As is well known, the phase unwrapping in optical interferometry is complicated by the unavoidable noise during the data acquisition process. Our strategy is to remove the noise by means of neural network curve fitting technique before performing phase unwrapping. The rest of the paper is organized as follows: Section II gives the background theory for OFSM system. The noise filtering and phase unwrapping method is proposed in Section III. Section IV and V presents the simulation and experimental result respectively. The conclusion is given in Section VI.

\section{BACKGROUND THEORY}

The widely accepted theoretical model for the OFSM system is known as Lang-Kobayashi equations [10], taking the forms as follows:

$$
\begin{aligned}
& \phi_{F}(t)=\phi_{0}(t)-C \cdot \sin \left[\phi_{F}(t)+\arctan (\alpha)\right] \\
& P(t)=P_{0}[1+m F(t)] \\
& F(t)=\cos \left(\phi_{F}(t)\right)
\end{aligned}
$$

Eq.(1) represents the phase condition where $\phi_{0}(t)$ and $\phi_{F}(t)$ are the laser phases without and with feedback respectively. $t=2 L / c$, is the round trip time between the $\mathrm{LD}$ and the external target. $\mathrm{C}$ is called feedback factor. Eq.(2) accounts for the laser power fluctuations as a result of the 
interferometric phase variations with $P(t)$ and $P_{0}$ denote the laser power with and without feedback respectively. $m$ is called modulation index (typically $\mathrm{m} \approx 10^{-3}$ ). $F(t)$ is defined by Eq.(3) and is a periodic function of period $2 \pi$.

Theoretically, the unperturbed laser phase $\phi_{0}(t)$ can be obtained from solving the three equations, once $P(t)$ is measured in an OFSM experimental setup. As $\phi_{0}(t)=2 \pi \cdot\left(2 L(t) / \lambda_{0}\right)$ where $L(t)$ is the target distance and $\lambda_{0}$ is the unperturbed laser wavelength, the target displacement (or moving track) can be retrieved by the relation $\Delta L(t)=L(t)-L_{0}=\phi_{0}(t) \lambda_{0} / 4 \pi-L_{0}$ with $L_{0}$ the initial target distance from the LD. However, when looking at Eq.(3) in more detail, one can find that $\phi_{F}(t)$ is computed from an inverse cosine function which always produces values in the interval $(-\pi, \pi]$. This is a common problem encountered in the optical interferometry and is termed wrapped phase. As a consequence, the technique to reconstruct the true phase map for the wrapped phase is called phase unwrapping which has been an active research topic for more than two decades with the outcome of numerous algorithms. Whereas the existent phase unwrapping algorithms are not ready to be used in this context due to the fact that the OFSM waveforms differ significantly from the others such as in SAR or fringe interferometry.

The basic mechanism for phase unwrapping is rather simple. Given the interferometric signal is sampled at a frequency much higher than the Nyquist rate according to the sampling theory, there is only small phase difference exists between any two adjacent points in the wrapped phase except those points with near $2 \pi$ phase jumps. Hence phase unwrapping can be carried out by successive comparison of the adjacent wrapped phases. Once a large phase jump is encountered, an integer multiple of $2 \pi$ should be added or subtracted [11].

In practice, the SM signals are always contaminated with random noises and speckles which renders phase unwrapping non-trivial. Intuitively if the noise is eliminated effectively, unwrapped phase could be readily achieved. As can be seen in the following text, a variety of data processing methods are proved necessary to remove different type of noises in the experimental data.

\section{NOISE FILTERING AND PHASE UNWRAPPING}

Our first attempts were made to filter the noise by traditional spatial and frequency filters. The phase field was first filtered by median filter to remove the speckles and then by mean filter to remove the white noise. The tradeoff in choosing the filter length is the ability to presume the characteristics of the signal and the noise removal performance. More filter points will yield smoother signal waveform which translates into lost signal property. Less filter points by contrast is capable of maintaining the signal characteristics with sacrifice of the noise removal performance. The empirical filter length is five in our case. Whereas in most circumstances the resulting waveform is far from satisfaction that can be used to perform unwrapping due to the complex nature of the noises. Recent development of neural network has recognized itself a powerful tool for pattern recognition and non-linear system modelling, which also sheds light on the noise elimination in SMS signal processing studies.

We explored the application of neural networks in order to remove the noises. The majority of neural networks are multilayer structures which consist of a layer of input neurons, a layer of output neurons and one or more layers of hidden neurons (so called as both their input and output are unknown to the user). The output is a nonlinear function of the input variables [12]. In this work, we employed a neural network with a single layer of hidden neurons with a sigmoid activation function and a linear output neuron. The output of the network is thus given by

$$
g(x, w)=\sum_{i=1}^{N_{c}}\left[w_{N_{c}+1, i} \tanh \left(\sum_{j=1}^{n} w_{i j} x_{j}+w_{i o}\right)\right]+w_{N_{c+1,0}}
$$

where $x$ is the input $(\mathrm{n}+1)$ vector, and $w$ is the vector of $(n+1) N_{c}+\left(N_{c}+1\right)$ parameters.

Both simulation and experimentation were carried out using the neural network toolbox for MATLAB. The training data are median filter- and mean filter-processed experimental SMS signals. Each training set consists of two hundred data points which were randomly fed to the network. The Root Mean Square (RMS) error between the desirable and actual output was calculated by the package. When the RMS error reached its convergence threshold which is set up by the user (in our case 0.01 ), the training was completed. We tested the experimental SMS signals at different feedback and found that the weak feedback curves can be fitted very well with the proposed network which yields best result with five neurons at the hidden layer. However, the situation for moderate feedback is a bit complex as the network does not perform good fitting for the curves with jumps. We solved this problem by segmenting the waveforms at the jumping points and fitted the signal segments only, satisfactory outcome were produced as a result. Curve fitted data are then extracted from the network as noise-filtered SMS data to recover the phase.

$\phi_{F}(t)$ was reconstructed from the above pre-processed self-mixing waveforms based on the following algorithm:

$$
\phi_{F}(t)=(-1)^{M_{1}} \arccos (F(t))+M_{2} \cdot 2 \pi
$$

Where $\mathrm{M}_{1}$ accumulated by one when the self-mixing signal reaches peak and valley points and $\mathrm{M}_{2}$ incremented by one when the SMS reaches the valleys. This is illustrated in Fig. 1. One fringe of the SMS corresponds to a $2 \pi$ changes in $\phi_{F}$. The starting point is selected where the target reaches the farthest position toward or from the LD, It is worth noting that in the proposed method the target moving law should be a known priori.

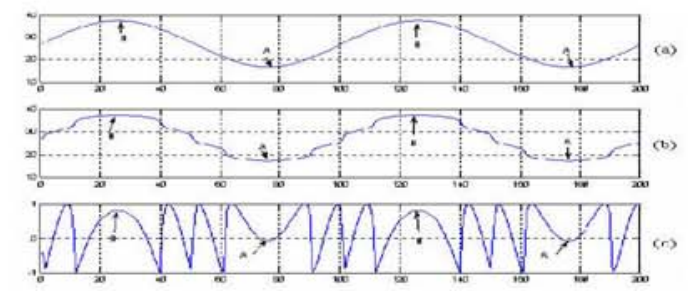

Fig. 1 Target moving track (a) $\phi_{0}$ (b) $\phi_{F}$ and SMS waveform (c). 


\section{SIMULATIONS}

The proposed phase unwrapping method was tested firstly with computer simulations. The external target is assumed to be subject to a harmonic vibration which can be represented as $L(t)=L_{0}+\Delta L \cos (2 \pi f)$, where $L_{0}$ is the initial distance between the laser front facet and the target, $f$ is the vibration frequency, $t$ is time variable. The laser phase without feedback is then calculated as

$$
\phi_{0}(t)=\frac{4 \pi L(t)}{\lambda_{0}}=\frac{4 \pi L_{0}}{\lambda_{0}}+\frac{4 \pi \Delta L}{\lambda_{0}} \cos (2 \pi f)
$$

If we assume $f=20 \mathrm{~Hz}, L_{0} / \lambda_{0}=20000$ and $\Delta L / \lambda_{0}=2$, the self-mixing signal can be generated using (1)-(3) and (6) with $C=0.8, \alpha=4$ for weak feedback and $C=3, \alpha=4$ for moderate feedback. A small white noise is also added with signal-to-noise ratio (SNR) of $20 \mathrm{~dB}$ to emulate the practical situation as shown in Fig. 2. The curve fitted waveforms are plotted in Fig. 3. In order to evaluate the accuracy of our method, we compare the reconstructed moving track with its real counterpart and the error is plotted in Fig. 4. It can be seen that accuracy of our proposed method can reach $\lambda / 25$ in the case of weak feedback and $\lambda / 20$ where moderate feedback is presented.
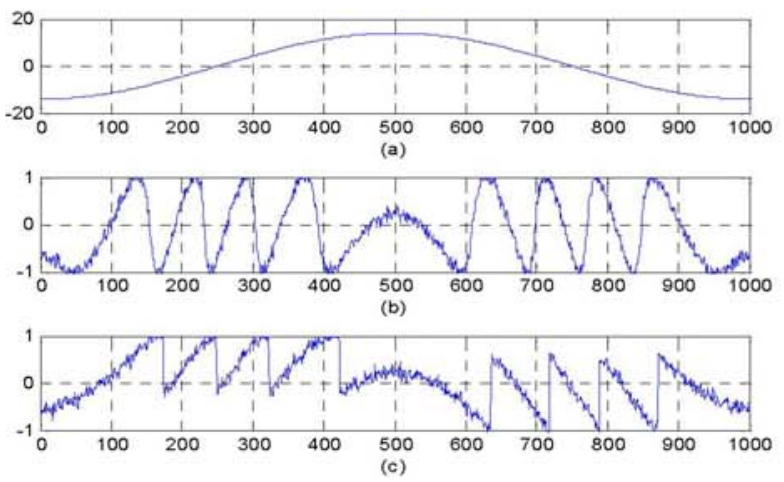

Fig. 2 Simulated SMS signals with $\mathrm{SNR}=20 \mathrm{~dB}$ (a) moving track of external target (b) SMS with $\mathrm{C}=0.8$ and $\alpha=4$ (c) SMS with $\mathrm{C}=3$ and $\alpha=4$

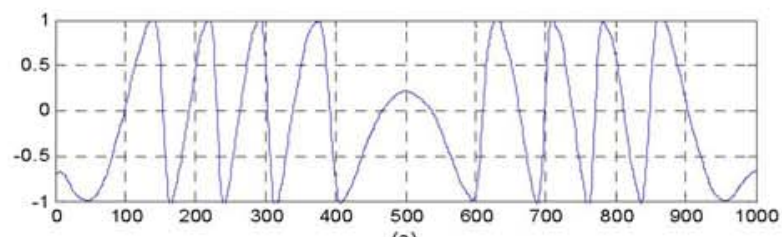

(a)

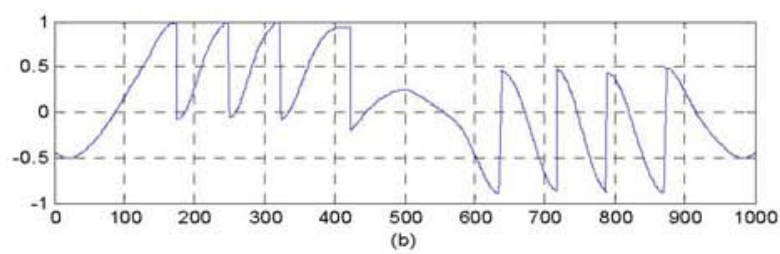

Fig. 3 Curve fitted SMS signals (a) Weak feedback (b) Moderate feedback
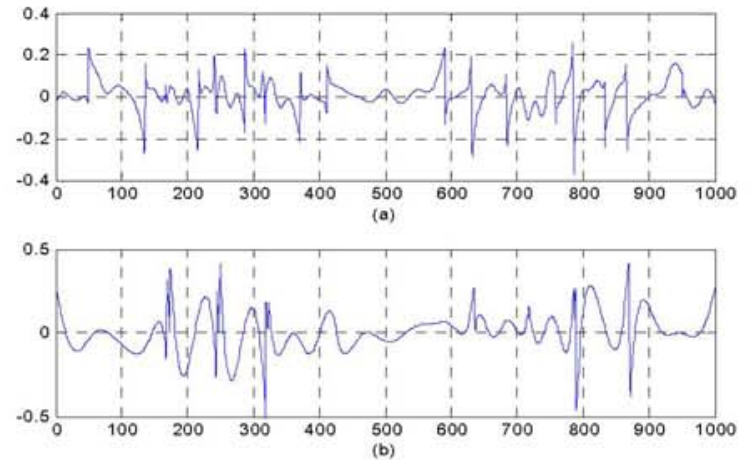

Fig. 4 The error between recovered target movement track and the real track (a) weak feedback (b) moderate feedback

\section{EXPERIMENT RESULTS}

In the OFSM experimental setup, the SL is biased with a dc current. A metal plate is used as the target, which is made to vibrate harmonically by placing it close to a loudspeaker driven by a sinusoidal signal. The SMS is detected by the monitor photodiode (PD) and is amplified by a transimpedance amplifier, as shown in Fig. 5 [5].

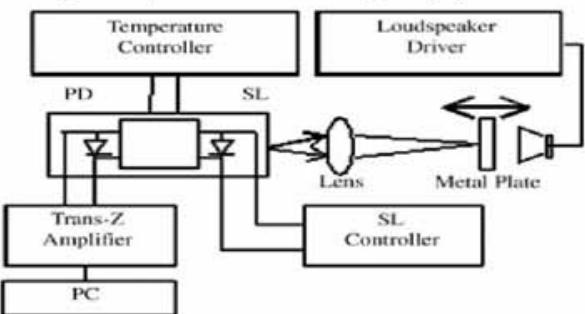

Fig. 5 OFSM experimental setup

The neural network training took approximately 1000 presentations of data to reach the convergence criterion. It was shown that testing waveforms are fitted very well with the neural network and the target moving tracks are recovered correctly based on the unwrapped phases. The waveforms of the SMS in weak feedback (up) and moderate feedback (down) are shown in Fig. 6. Speckles and white noised are significant in both signals.

Fig. 7-a, b show the median filter and mean filter processed one period SMS signal of the harmonic movement of the target in weak feedback and moderate feedback respectively. It can be found that the random noise present in the moderate feedback SMS is still significant. Fig. 7-c, d shows further neural network processed SMS signals for weak feedback and moderate feedback circumstances respectively. It is clearly seen that the noises have been effectively eliminated.

Fig. 8 shows the target moving track recovered from SMS waveforms. It is approximately harmonic, which reveals a good recovery of the real movement of the target. The speckles along the recovered moving track for moderate feedback is due to the broadened jumping sections in the measured SMS waveforms.

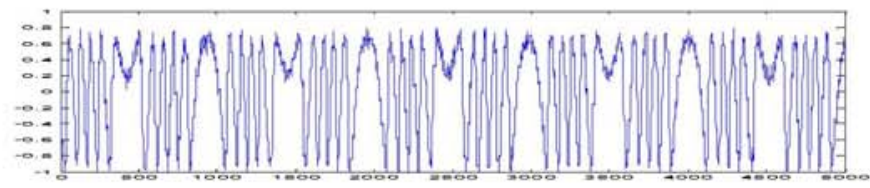

(a) 


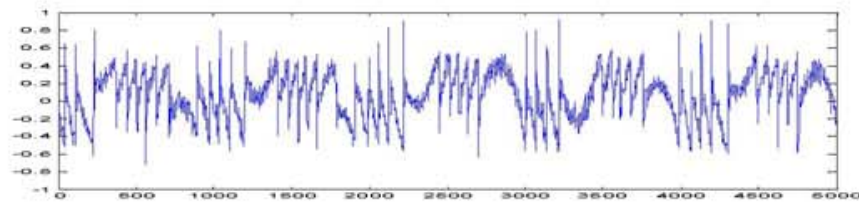

(b)

Fig. 6 Experimental SMS waveforms for

(a) weak feedback and (b) moderate feedback

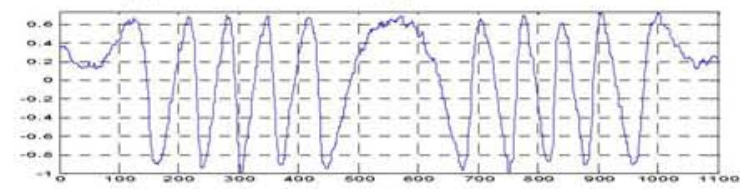

(a)

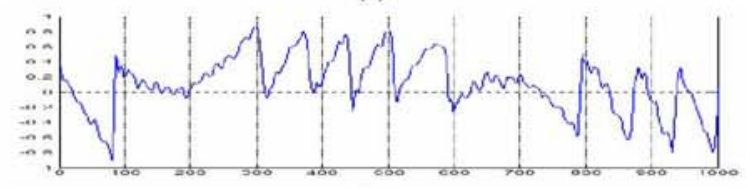

(b)

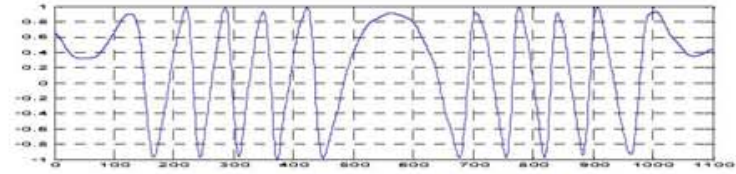

(c)

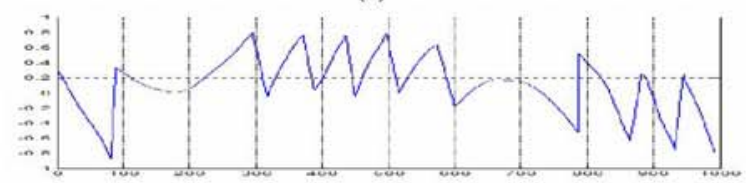

(d)

Fig. 7 SMS waveforms before and after neural network curve fitting.

(a) Median filter and mean filter processed SMS signals for weak feedback

(b) Median filter and mean filter processed SMS signals for moderate

feedback (c) Neural network processed SMS signals for weak feedback

(d) Neural network processed SMS signals for moderate feedback

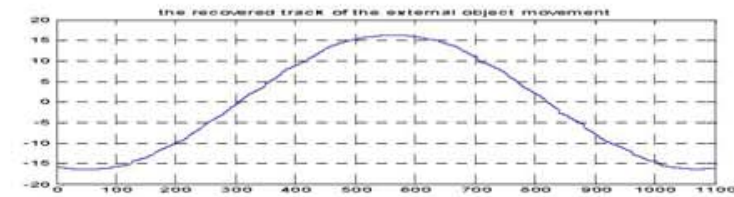

(a)

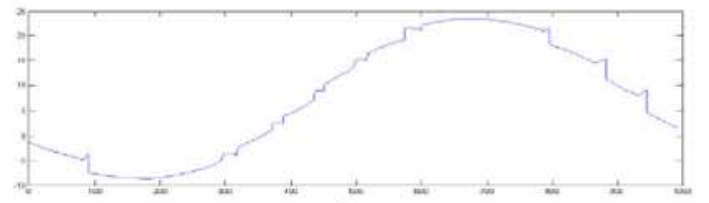

(b)

Fig. 8 Recovered target moving track

(a) weak feedback (b) moderate feedback

\section{CONCLUSION}

A phase-unwrapping based displace measurement approach is proposed in this paper. Neural network curve fitting method has been employed to remove the noises in SMS signals after median and mean filtering. The fitting results show effective elimination of noises while maintain good coordination between the input data and the fitted curve. With the proposed algorithm the phases with optical feedback have been unwrapped and the target moving trajectory has recovered with good accuracy of $\lambda / 25$ for weak feedback and $\lambda / 20$ for moderate feed back according to computer simulation.

\section{References:}

[1] P. A. Roos, M. Stephens, and C. E. Wieman, "Laser vibrometer based on optical-feedback induced frequncy modulation of a single-mode laser diode," Applied Optics, vol. 35, pp. 6754-6761, December, 1996.

[2] S. Donati, G. Giuliani, and S. Merlo, "Laser diode feedback interferometer for measurement of displacements without ambiguity," Quantum Electronics, IEEE Journal of, vol. 31, pp. 113-119, 1995.

[3] G. Giuliani, M. Norgia, S. Donati, and T. Bosch, "Laser diode self-mixing technique for sensing applications," Journal of Optics A: Pure and Applied Optics, vol. 4, pp. S283-S294, 2002.

[4] L. Scalise, Y. G. Yu, G. Giuliani, G. Plantier, and T. Bosch, "Self-ming laser diode velocimetry: Application to vibration and velocity measurement," IEEE Trans. Instrum. Meas., vol. 53, pp. 223-232, Feb., 2004.

[5] J. Xi, Y. Yu, J. F. Chicharo, and T. Bosch, "Estimating the parameters of semiconductor lasers based on weak optical feedback self-mixing interferometry," Quantum Electronics, IEEE Journal of, vol. 41, pp. 1058-1064, 2005.

[6] R. C. Addy, A. W. Palmer, and K. T. V. Grattan, "Effects of external reflector alignment in sensing applications of optical feedback in laser diodes," Journal of Lightwave Technology, vol. 14, pp. 2672-2676, Dec, 1996.

[7] T. Bosch and N. Servagent, "A displacement sensor for spectrum analysis using the optical feedback in a single-mode laser diode," presented at IEEE instrumentation and measurement technology conference, Ottawa, Canada, May, 1997.

[8] N. Servagent, F. Gouaux, and T. Bosch, "Measurements of displacement using the selfmixing interference in a laser diode," J. Opt., vol. 29, 1998.

[9] F. Gouaux, N. Servagent, and T. Bosch, "A phasemodulated method to improve the resolution of a self-mixing interferometer," presented at IEEELEOS ODIMAP II, Pavia, May 1999.

[10] R. Lang and K. Kobayashi, "External optical feedback effects on semiconductor injection laser properties," Quantum Electronics, IEEE Journal of, vol. 16 , pp. $347-355,1980$.

[11] D. W. Robinson, "Phase unwrapping methods," in Interferogram Analysis; Digital Fringe Pattern Measurement Techniques, D. W. Robinson and G. T. Reid, Eds. Philadelphia, PA: Inst. Physics, 1993, pp. 194-228.

[12] G. Dreyfus, Neural networks: Methodology and applications. Berlin, Germany: Springer-Verlag, 2005. 